\title{
INFLUENCIA DA SECA PROLONGADA NA CONCENTRAÇÃO DE SÓLIDOS TOTAIS E NA SALINIDADE DE ÁGUAS DA BACIA HIDROGRÁFICA DO RIO APODI/MOSSORÓ
}

Natália Cristiane Gomes Da Silva ${ }^{1}$ (Graduanda em Química na Universidade do Estado do Rio Grande do Norte - UERN);

Luiz Di Souza²(Prof. Adjunto do Dq e do PPGCN na Universidade do Estado do Rio Grande do Norte - UERN);

Jesyka Macedo Guedes (Graduanda em Química na Universidade do Estado do Rio Grande do Norte - UERN);

Ewelanny Louyde Ferreira Limaª(Graduanda em Química na Universidade do Estado do Rio Grande do Norte - UERN).

*E-mail: natalia-cgs@hotmail.com

Resumo: A água é um recurso natural primordial, como componente bioquímico de seres vivos, como fonte de vida de várias espécies vegetais e animais, como parte representativa de valores sociais e culturais e como fator de produção de bens de consumo. A água do planeta é distribuída em oceanos, geleiras e calotas polares, águas subterrâneas, lagos, mares, umidade do solo, atmosfera e rios. A água existe em grande quantidade, mas está mal distribuída, existindo em abundância em alguns locais pouco povoado e em pouca quantidade em locais muito povoados e com alta necessidade. Para cumprir suas funções a água precisa estar disponível em quantidade e com qualidade especifica para cada uso, sendo essa um fator que é negligenciado pela sociedade e está diminuindo a disponibilidade de água potável no mundo. Além disso, algumas regiões enfrentam periodicamente condições naturais que alteram a quantidade e a qualidade da água numa determinada região, as chamadas secas. O Nordeste brasileiro está passando no momento pela sua seca mais prolongada, sendo grande e amplamente divulgada a falta de água, no entanto sobre a qualidade da água ainda existente pouco se sabe. Assim o objetivo do presente trabalho foi verificar a qualidade da água comparando resultados de alguns parâmetros no ano de 2011 e do ano de 2015. Foram analisado os parâmetros de sólidos totais e salinidade de vários reservatórios de água do estado do Rio Grande do Norte se avaliando além da sazonalidade a sua localização (espacialidade) e comparando os mesmos com a legislação. Os resultados mostraram que a longa estiagem está afetando fortemente a qualidade da água e que este fator é agravado pelo fator localização do recurso hídrico, sendo os mais próximos de áreas densamente povoadas e, portanto, mais sujeitas a poluição antrópica os mais afetados e poluídos.

\section{Palavras chaves:}

Água; Salinidade; Sólidos Totais; Nordeste.

\section{1- Introdução}

A água é um recurso fundamental para a existência da vida. Foi na água que a vida floresceu, e seria difícil imaginar a existência de qualquer forma de vida na ausência deste recurso vital 
(GRASSI, 2001). A água é essencial para a vida da maior parte dos organismos vivos e excelente solvente para muitas outras substâncias. Segundo Martins, et al (2007), este recurso já foi considerado inesgotável e é visto nos dias atuais como fator estratégico de desenvolvimento, a ponto de ser qualificada pela ONU como "petróleo do século XXI", Isto se deve aos problemas de falta de gerenciamento adequado do uso, a contaminação dos corpos hídricos e mananciais, e principalmente, a sua distribuição desigual. A água do planeta é distribuída em oceanos, geleiras e calotas polares, águas subterrâneas, lagos, mares, umidade do solo, atmosfera e rios (GRASSI, 2001). Existe cerca de $71 \%$ da superfície da terra coberta por água. De acordo com Clarke e King (2005), cerca de 97,4\% dessa água apresenta-se espalhada por oceanos, mares, lagos salgados e aquíferos salinos e somente uma pequena fração dessa imensa quantidade, 2,6\%, corresponde à água doce. Vale ressaltar que, dessa última, apenas uma parcela menor ainda apresenta-se na forma de água doce acessível para atender as necessidades atuais.

Porém esse não é o caso da região do nordeste brasileiro como mostra a Agência Nacional de Águas (ANA) que comprova que o Nordeste possui a menor disponibilidade hídrica do país. Ou seja, 3\% (PAZ, 2013). O Nordeste do Brasil situa-se entre as latitudes $1^{\circ}$ e $18^{\circ} 30^{\prime} \mathrm{S}$ e as longitudes $34^{\circ} 30^{\prime}$ e $40^{\circ} 20^{\prime} \mathrm{W}$ e ocupa a área de $1.219 .000 \mathrm{~km}^{2}$, que equivalem a aproximadamente um quinto do território brasileiro. A região abrange os estados do Maranhão, Piauí, Ceará, Rio Grande do Norte, Paraíba, Pernambuco, Alagoas, Sergipe e Bahia, nos quais vivem 18,5 milhões de pessoas e dos quais 8,6 milhões estão na zona rural (CIRILO et al, 2007). Dentre os muitos aspectos apresentados pela região o que mais se destaca é a seca, causada pela escassez de chuvas, fenômeno natural que ocorre em alguns anos. No caso, atualmente a região passa pela pior seca de sua história como mostram os dados da figura1 para todo o nordeste brasileiro.

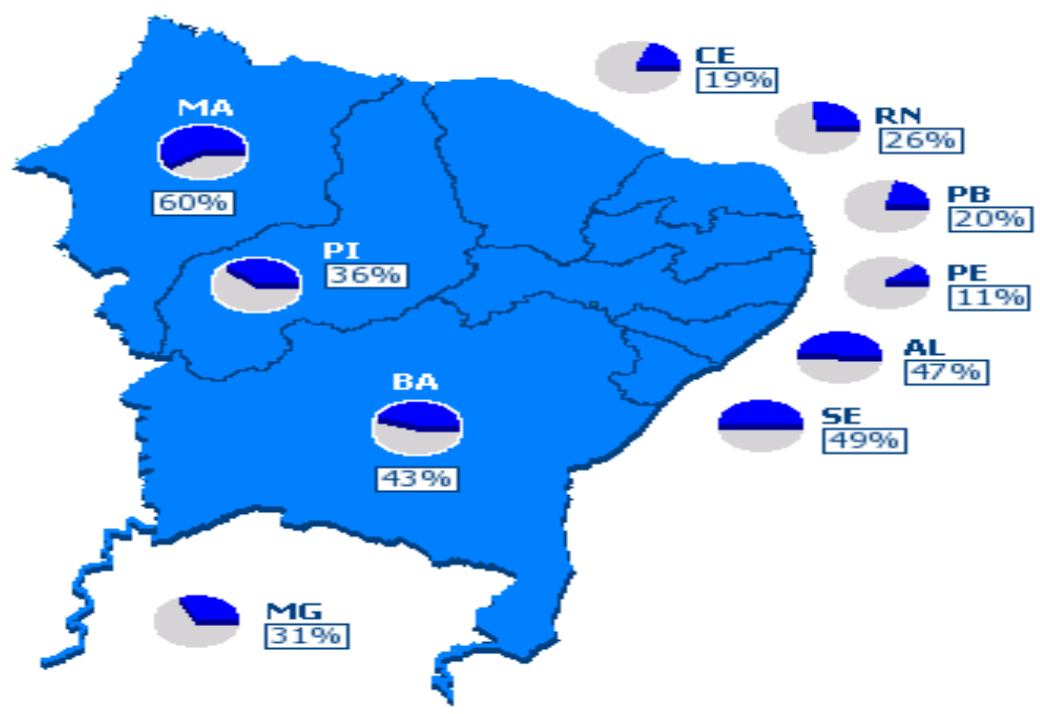

Figura 1. Resumos dos recursos hidricos disponiveis no nordeste em setembro de 2015. Fonte: Site DNOCS. 
Na região Oeste potiguar área deste trabalho a situação é a mesma como ilustra o gráfico 1 do volume acumulado na barragem Armando Ribeiro Gonçalves, o maior reservatório hídrico da região.

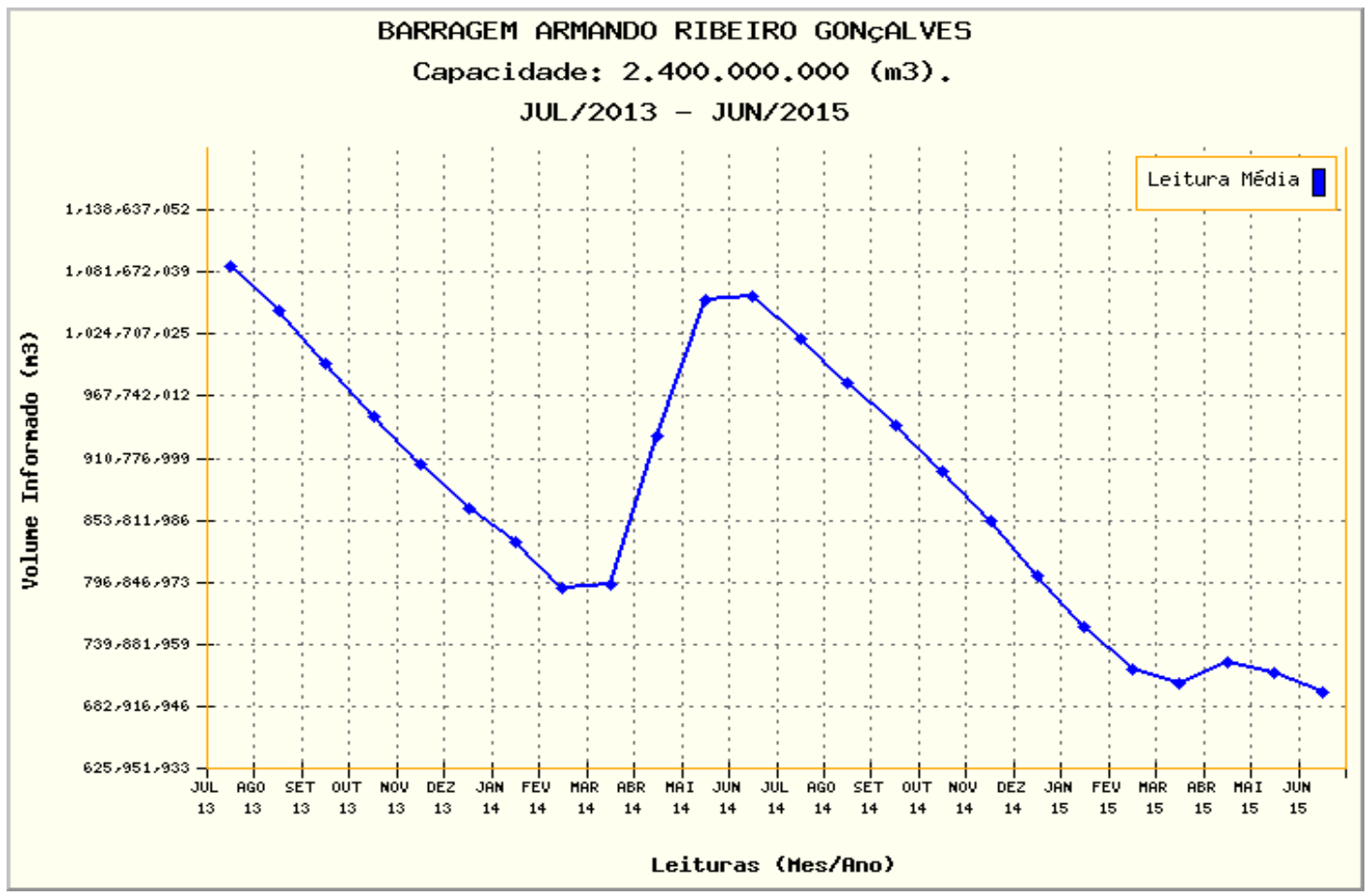

Gráfico 1. Volume $\left(\mathrm{m}^{3}\right)$ da barragem Armando Ribeiro Gonçalves nos anos de 2013 - 2015. Fonte: Site DNOCS.

Devido a essa escassez de água, especialmente neste momento em que se passa pela pior seca da história (SAKAUE 2015), a preocupação tem aumentado e tem-se procurado formas para preservação desse bem. No nordeste devido a suas condições especificas de baixa pluviosidade e longos períodos sem chuva é comum a construção de reservatórios (barragens, açudes, barreiros, cisternas...) para estocar água nos períodos de estiagem.

A escassez de água pode acarretar diversos problemas como a contaminação dos rios, período de plantação e colheita alterado, diminuição na produção industrial, danos ambientais e doenças (hepatite, febre tifoide, diarreia infantil, cólera) (CANOBEL, 2009). Tendo em vista todos os problemas causados pela contaminação das águas, se faz necessário avaliar como anda a qualidade da água em alguns reservatório. Dessa forma tivemos como objetivo coletar e analisar os parâmetros de sólidos totais e salinidade de pontos do Rio Apodi/Mossoró nos anos de 2011 e 2015 com o intuito de comparar os danos causados pela seca enfrentada pela região que já se estende a 5 anos.

\section{2- Material e Métodos}

\section{1- Coletas}

As amostras foram coletadas conforme orientações prescritas na NBR 9897(ABNT, 1987; (Planejamento de amostragem de efluentes líquidos e corpos receptores) e preservadas de acordo com a NBR 9898 (ABNT, 1987; Preservação e técnicas de amostragem de efluentes líquidos e corpos receptores), transportadas e analisadas de acordo com as metodologias prescritas no Standard Methods for Examination of Water and Wastewater (APHA, 2005). 
Os parâmetros (Salinidade e ST) foram quantificados utilizando-se da estrutura do Laboratório de Catálise, Ambiente e Materiais (LACAM), pertencente a Universidade do Estado do Rio Grande do Norte - UERN. Todas as análises foram feitas em triplicata e a média aritmética das medidas foi considerada como sendo o resultado das mesmas.

\section{2- Caracterização da Área de Estudo}

O rio Apodi/Mossoró é o segundo maior rio potiguar, com cerca de $210 \mathrm{~km}$ de extensão. Nasce na Serra de Luís Gomes, passa pelos municípios localizados na chapada do Apodi e, depois de banhar a cidade de Mossoró, deságua no Oceano Atlântico, entre os municípios de Grossos e Areia Branca, onde se situam grandes salinas. Na margem direita, o rio Mossoró tem como afluentes os rios Carmo-Upanema, Umari e Pitombeira; na margem esquerda, os rios Apodi, Tapuio, Grande e Bom Sucesso. O rio só mantém sua perenização no baixo-curso, pois é alimentado por fontes d'água que escorrem das partes altas da chapada do Apodi e, principalmente, pela barragem de Santa Cruz no município de Apodi e por outras menores construída em seu leito, já nas proximidades e na cidade de Mossoró.

O Rio Apodi apresenta-se altamente poluído por lixo e esgoto urbano, principalmente no trecho a partir da cidade de Mossoró. O Rio passa pelos municípios de Luiz Gomes, Pau dos Ferros, Itaú, Apodi, Felipe Guerra, Mossoró, Areia Branca, entre outros, servindo a comunidade e recebendo os seus efluentes. O percurso da Bacia Hidrográfica do Rio Apodi/Mossoró é mostrado na figura 2 abaixo.

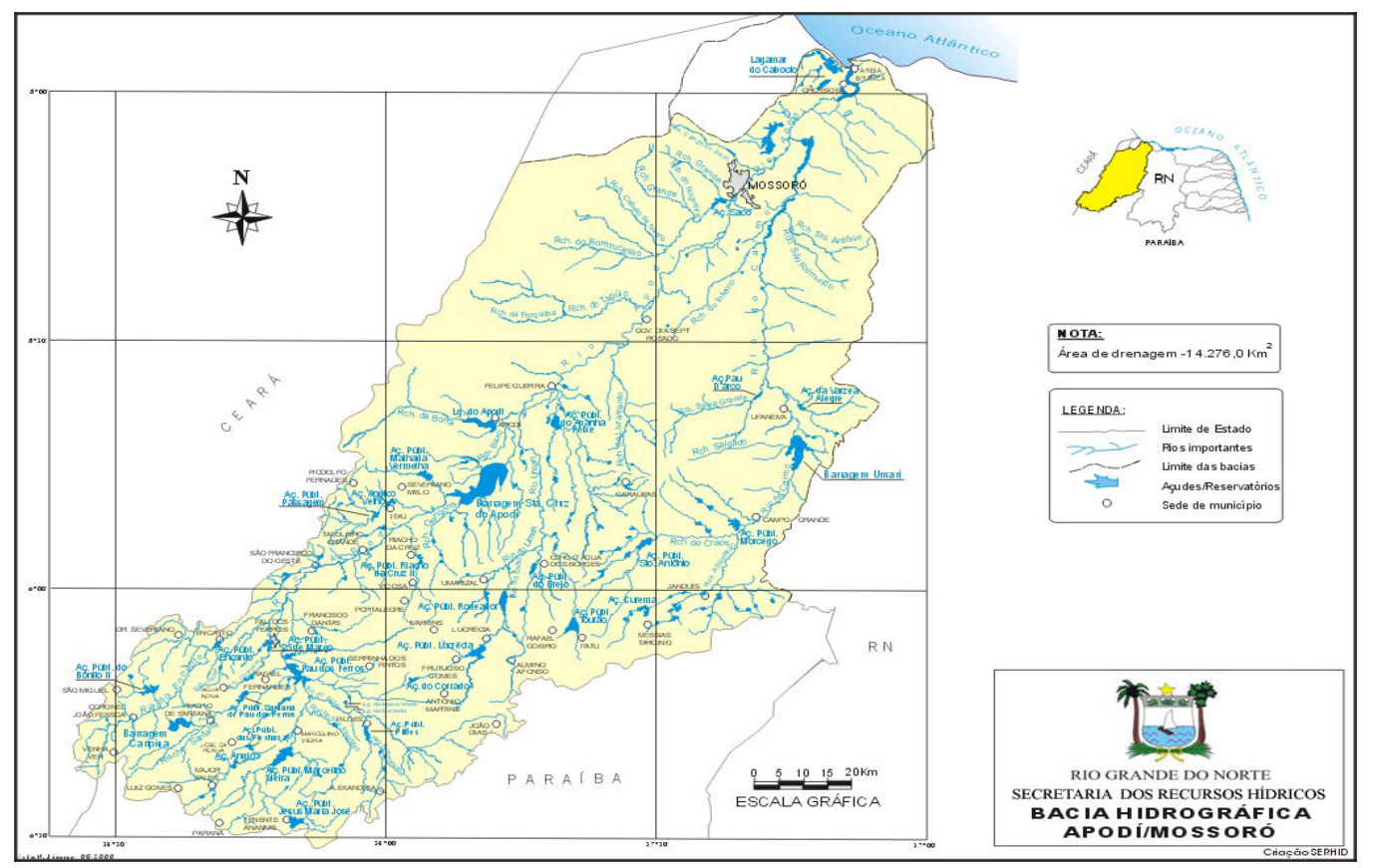

Figura 2. Localização da Bacia Hidrográfica do Apodi-Mossoró no estado do Rio Grande do Norte. (Fonte: SERHID 2003).

\section{3- Resultados e Discussões}

A tabela apresenta os resultados obtidos para a salinidade e RST nos anos de 2011 e 2015 em alguns locais da bacia hidrográfica do rio Apodi/Mossoró.

Tabela 1- Resultados dos parâmetros físico-químicos analisados e seus limites no CONAMA. 


\begin{tabular}{|c|c|c|c|c|c|c|}
\hline $\begin{array}{l}\text { Pontos } \\
\text { analisados }\end{array}$ & ST 2011 & ST 2015 & $\begin{array}{c}\text { CONAMA } \\
\text { ST }\end{array}$ & $\begin{array}{l}\text { SALINID } \\
\text { ADE } 2011\end{array}$ & $\begin{array}{l}\text { SALINIDA } \\
\text { DE } 2015\end{array}$ & $\begin{array}{l}\text { CONAMA } \\
\text { Salinidade }\end{array}$ \\
\hline APM 1 (A.E.) & 486 & 220 & $500 \mathrm{mg} / \mathrm{L}^{-1}$ & 1318 & 23,072 & $412 \mathrm{mg} / \mathrm{L}^{-1}$ \\
\hline APM 2 (A.B. II) & 386 & 1090 & $500 \mathrm{mg} / \mathrm{L}^{-1}$ & 161,50 & 184,56 & $412 \mathrm{mg} / \mathrm{L}^{-1}$ \\
\hline APM 4 (A.F.) & 278 & 660 & $500 \mathrm{mg} / \mathrm{L}^{-1}$ & 92,29 & 369,15 & $412 \mathrm{mg} / \mathrm{L}^{-1}$ \\
\hline APM 5 (B.P.F.) & 240 & 1222 & $500 \mathrm{mg} / \mathrm{L}^{-1}$ & 69,22 & 380,68 & $412 \mathrm{mg} / \mathrm{L}^{-1}$ \\
\hline APM & 204 & 546 & $500 \mathrm{mg} / \mathrm{L}^{-1}$ & 46,14 & 207,65 & $412 \mathrm{mg} / \mathrm{L}^{-1}$ \\
\hline APM 9 (A.L.) & 322 & 956 & $500 \mathrm{mg} / \mathrm{L}^{-1}$ & 92,29 & 530,66 & $412 \mathrm{mg} / \mathrm{L}^{-1}$ \\
\hline APM 10 (A.R.) & 224 & 436 & $500 \mathrm{mg} / \mathrm{L}^{-1}$ & 69,22 & 184,57 & $412 \mathrm{mg} / \mathrm{L}^{-1}$ \\
\hline APM 12 (M.V.) & 488 & 7.908 & $500 \mathrm{mg} / \mathrm{L}^{-1}$ & 323,01 & 4.130 & $412 \mathrm{mg} / \mathrm{L}^{-1}$ \\
\hline APM 13 (A.P.) & 136 & 270 & $500 \mathrm{mg} / \mathrm{L}^{-1}$ & 23,07 & 103,82 & $412 \mathrm{mg} / \mathrm{L}^{-1}$ \\
\hline APM 14 (A.M.) & 48 & 1222 & $500 \mathrm{mg} / \mathrm{L}^{-1}$ & 23,7 & 115,36 & $412 \mathrm{mg} / \mathrm{L}^{-1}$ \\
\hline APM 15(A.S.A.) & 162 & 3.428 & $500 \mathrm{mg} / \mathrm{L}^{-1}$ & 46,14 & 162 & $412 \mathrm{mg} / \mathrm{L}^{-1}$ \\
\hline APM & 360 & 876 & $500 \mathrm{mg} / \mathrm{L}^{-1}$ & 92,29 & 277 & $412 \mathrm{mg} / \mathrm{L}^{-1}$ \\
\hline APM 20 (B.G.) & 502 & 2020 & $500 \mathrm{mg} / \mathrm{L}^{-1}$ & 207,65 & 1384 & $412 \mathrm{mg} / \mathrm{L}^{-1}$ \\
\hline APM 21 (P.P.) & 838 & 1638 & $500 \mathrm{mg} / \mathrm{L}^{-1}$ & 238,37 & 1073 & $412 \mathrm{mg} / \mathrm{L}^{-1}$ \\
\hline APM 22 (A.B.) & 27.292 & 46.478 & $500 \mathrm{mg} / \mathrm{L}^{-1}$ & $2.1918,4$ & 39.222 & $412 \mathrm{mg} / \mathrm{L}^{-1}$ \\
\hline APM22(R.C.F. & 186 & 280 & $500 \mathrm{mg} / \mathrm{L}^{-1}$ & 92,29 & 323 & $412 \mathrm{mg} / \mathrm{L}^{-1}$ \\
\hline APM 24 (B.U.) & 104 & 270 & $500 \mathrm{mg} / \mathrm{L}^{-1}$ & 23,7 & 69 & $412 \mathrm{mg} / \mathrm{L}^{-1}$ \\
\hline APM & 140 & 276 & $500 \mathrm{mg} / \mathrm{L}^{-1}$ & 46,14 & 115 & $412 \mathrm{mg} / \mathrm{L}^{-1}$ \\
\hline APM 27 (А.Т.) & 316 & 3.782 & $500 \mathrm{mg} / \mathrm{L}^{-1}$ & 138,43 & 1690,79 & $412 \mathrm{mg} / \mathrm{L}^{-1}$ \\
\hline APM30(P.BR11 & 626 & 43.050 & $500 \mathrm{mg} / \mathrm{L}^{-1}$ & 323.01 & 24.226 & $412 \mathrm{mg} / \mathrm{L}^{-1}$ \\
\hline
\end{tabular}

De acordo com os dados mostrados na tabela 1 e gráfico 2, considerando um erro experimental de $1 \%$, todos valores de sólidos totais no ano de 2011 estavam todos dentro do limite previsto, exceto os pontos, 21, 22 e 30 (15\%). 


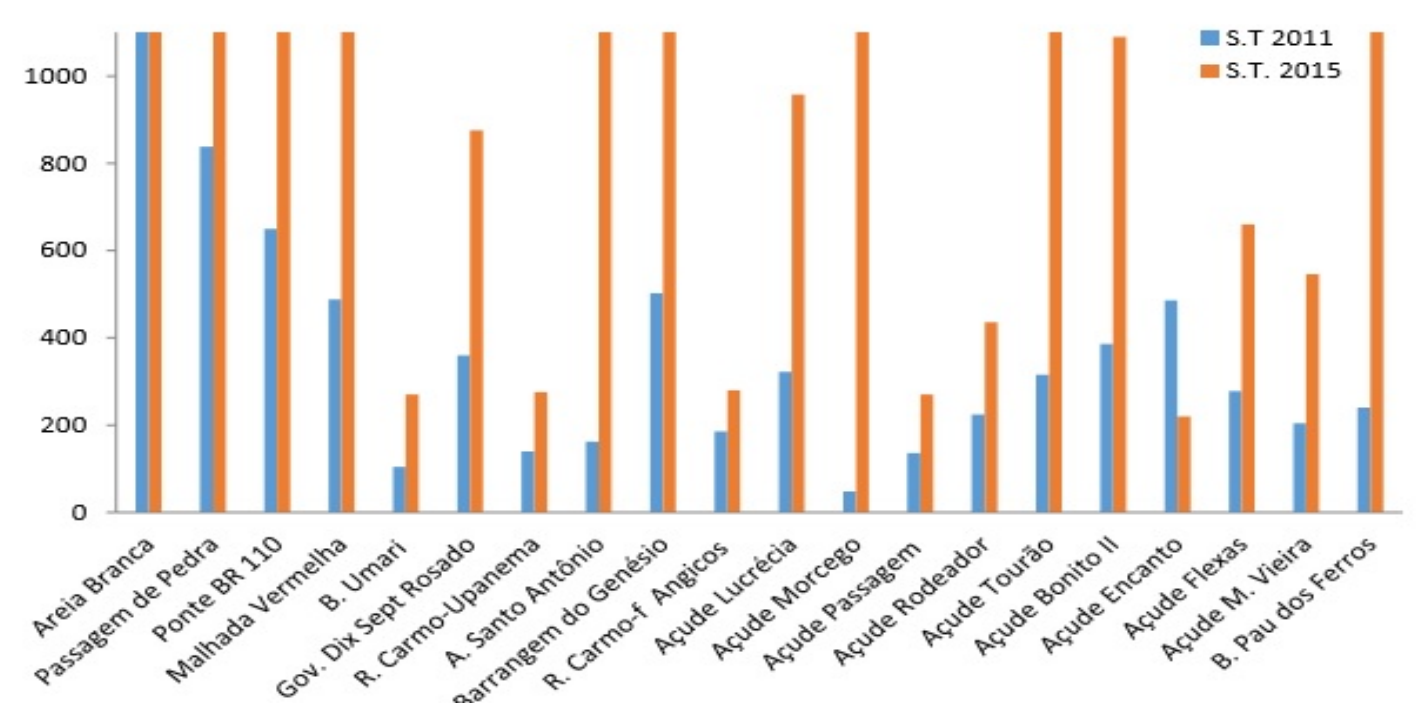

Gráfico 2- Teores de ST correspondentes aos anos de 2011 e 2015. (Autoria própria 2015)

O mesmo fenômeno é encontrado nas análises de salinidade (tabela 1 e gráfico 3).

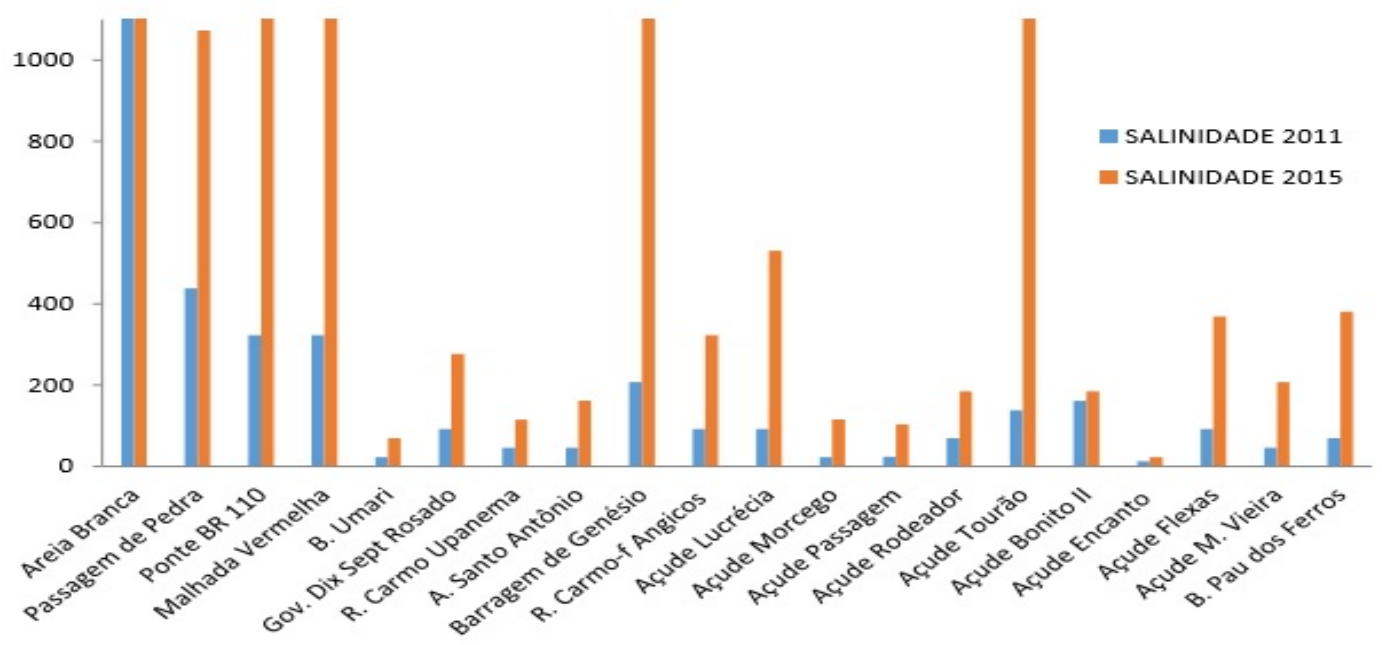

Gráfico 3- Teores de salinidade nos anos de 2011 e 2015. (Autoria própria)

Sendo que essas não refletem o resultado da presença de sólidos orgânicos na água e sim a presença dos sais inorgânicos dissolvidos. Neste caso 35 \% dos pontos estão acima do limite estabelecido em 2015 em comparação com apenas 10 \% que estavam acima em 2011, cabendo ressaltar que um dos pontos acima do limite é o porto de Areia Branca e portanto não se enquadra na resolução que é para água doces e o outro é um pequeno açude na região serrana, o qual pode ter recebido alguma chuva não esperada que alterou os seus resultados. No ano de 201513 pontos (65\%) estão com os valores de salinidade acima do permitido, cabendo ressaltar que as concentrações excedem em muito o limite estabelecido.

A explicação para os pontos acima dos limites, com exceção do açude encanto já explicado por chuvas não esperadas:

a) É o fato de alguns pontos se encontrarem numa região de estuário (ponto 1,2 e 3) e serem influenciados pelas marés altas que invadem o rio e aumentam a quantidade de sais nas águas, como confirmam os resultados de salinidade. Além disso a região desses pontos é marcada por uma forte atividade salineira que descarrega os seus efluentes nesses pontos o que também contribui para aumentar as concentrações de sais na água. 
b) A conjugação dos efeitos das altas temperaturas e escassez de chuvas o que faz com que quantidade maior de água seja evaporada e a concentração de ST aumente em relação a menor quantidade de água que resta no reservatório, fato que vai se agravando progressivamente até ocorrerem chuvas que voltem a diluir esses sólidos, diminuindo sua concentração na água.

Estes resultados mostram que a longa estiagem está tendo um forte efeito sazonal na qualidade da água e que esta não está apropriada para alguns usos como, dessedentação (humana ou animal), conservação da fauna e flora. Irrigação e conservação da paisagem.

\title{
4- CONCLUSÃO
}

De acordo com os resultados, pode-se constatar que os quatro anos de seca (fator natural sazonal) alteraram muito a concentração de ST e salinidade dos pontos analisados. Com respeito a legislação 65 \% dos valores de St e 35 dos valores de salinidade se encontram em 2015 fora dos limites estabelecidos pela CONAMA e, portanto, inadequados para uma série de usos.

\begin{abstract}
:
Water is a vital natural resource, as biochemical component of living organisms, as a source of life of several plant and animal species, as part of social and cultural values and as a factor of production of consumer goods. The planet's water is distributed in oceans, glaciers and icecaps, ground water, lakes, seas, soil moisture, air and rivers. Water exists in large quantity, but is poorly distributed, and in abundance in some little town and in small amount in well-populated locations and with high need. To fulfill its functions the water needs to be available in quantity and with quality specifies for each use, a factor that is overlooked by society and is reducing the availability of potable water in the world. In addition, some regions face periodically natural conditions that alter the quantity and quality of water in a particular region, the so-called dry. The Brazilian Northeast is passing at the time for his prolonged drought, being large and widely disseminated the lack of water, however on water quality existing still little is known. So the purpose of this study was to verify the quality of the water by comparing results of some parameters in the year 2011 and the year 2015. Were analyzed the parameters of solids and salinity of several water reservoirs of the State of Rio Grande do Norte if assessing beyond the seasonality of its location (spatiality) and compare the same with the legislation. The results showed that the long drought is affecting the water quality and that this factor is exacerbated by water resource location factor, being the closest to densely populated areas, and thus more subject to anthropogenic pollution the most affected and polluted.
\end{abstract}

\section{5- Agradecimentos}

Agradecemos ao projeto água azul pela ajuda financeira e pelas coletas realizadas, ao laboratório de catálise, ambiente e materiais (LACAM), a Universidade do Estado do Rio Grande do Norte - UERN.

\section{6- REFERÊNCIAS}

APHA/AWWA/WEF. 2005. Standard methods for examination of water and wastewater. American Public Health Association, American Water Works Association and Water Environment Federation. 21st ed., Washington, USA. 4358 pp.Associação Brasileira de Normas Técnicas. ABNT. Preservação e técnicas de amostragem de efluentes líquidos e corpos receptores, 1987.

BRASIL. Agência Nacional de Águas. Panorama da qualidade das águas superficiais do Brasil: 2012. Agência Nacional de Águas - Brasília: ANA, 2012. 264 p. 
CANOBEL, A. Doenças e mortes causadas pela água poluída, falta de água e falta de saneamento básico; Março de 2009.subterrâneas. In: Cirilo, J.A., Cabral, J.J.S.P., Ferreira, J.P.L., Oliveira, M.J.P.M., Leitão, T.E., Montenegro, S.M.G.L. \& Góes, V.C. (orgs.). O uso sustentável dos recursos hídricos em regiões semi-áridas. ABRH,Editora Universitária da Universidade Federal de Pernambuco. p. 167-175.

SOUZA, L.D.; CASTRO, S. S. L., MARTINS, D. F. F.; OLIVEIRA, T. M. B. F.; Qualidade físico-química das águas da bacia do rio Apodi/Mossoró: I-Variabilidade espacial; Revista Química no Brasil, Campinas, SP: Editora Átomo, 2007, v.1, n. 1, jan/jun. 2007, p. 61.

CIRILO, J.A., Góes, V.C. \& Asfora, M.C. 2007. Integração das águas superficiais

CLARKE, R. e KING, J. (2005). O atlas da água: o mapeamento completo do recurso mais precioso do planeta. $1^{\circ}$ edição. Editora Publifolha. São Paulo.

GRASSI, M. T. Águas do planeta terra, Maio 2011.

Imagem, Departamento Nacional de Obras Contra as Secas. DNOCS. Disponível em:

$<$ http://www.dnocs.gov.br/dnocs/php/comunicacao/monitoramento_de_reservatorios.php>. Acesso em 22 Set. 2015.

PAZ, J. Nordeste tem apenas 3\% de agua doce no brasil; Meio Ambiente; Outubro, 2013

RICARDO, B. Água o risco da escassez; Socioambiental; Março, 2005.

SAKAUE. B. Seca que atinge o Nordeste chega ao grandes centros urbanos, G1 Jornal Hoje; Junho, 2015. 